\title{
Safety of early oral feeding after total laparoscopic radical gastrectomy for gastric cancer (SOFTLY-I): a single-center randomized controlled trial
}

This article was published in the following Dove Press journal: Cancer Management and Research

\author{
Quan Wang ${ }^{1,2}$ \\ Ke-Lu Yang ${ }^{1,3,4}$ \\ Bo-Yang Guo' \\ Li-Feng Shang' \\ Zun-Dong Yan' \\ Juan $\mathrm{Yu}^{\prime}$ \\ Di Zhang' \\ Gang Ji'
}

'Department of Digestive Surgery, Xijing Hospital of Digestive Disease, Xijing Hospital, The Fourth Military Medical University, Xi'an 710032, People's Republic of China; ${ }^{2}$ Department of Gastrointestinal Surgery \& Laboratory of Surgical Oncology, Peking University People's Hospital, Peking University, Beijing 100044, People's Republic of China; ${ }^{3}$ Evidence-Based Nursing Center, School of Nursing, Lanzhou University, Lanzhou 735000, People's Republic of China; ${ }^{4}$ Key Laboratory of Evidence Based Medicine and Knowledge Translation of Gansu Province, Lanzhou University, Lanzhou, 735000, People's Republic of China
Correspondence: Gang Ji

Department of Digestive Surgery, Xijing Hospital of Digestive Disease, Xijing Hospital, The Fourth Military Medical University, Xi'an, 710032, People's Republic of China

Email jigang@fmmu.edu.cn
Objectives: The aim of this study is to explore the safety and feasibility of early oral feeding (EOF) on short-term postoperative outcomes.

Trial design: A prospective randomized non-inferiority trial.

Materials and methods: From August 27, 2015 to March 31, 2017, 100 consecutive patients with gastric cancer in Xijing Hospital were recruited. Patients undergoing total laparoscopic radical gastrectomy (TLRG) received either EOF group or delayed oral feeding (DOF group). The endpoints were anastomotic leakage, the recovery of bowel function, the postoperative complications and costs. The process of randomization used a computergenerated sequence that was kept in a sealed envelope by a nurse that did not participate in the trial. None of the participants, administrators of interventions and those assessing outcomes was blinded.

Results: Ultimately, 51 patients were in EOF group and 49 in DOF group, which both are comparable. The postoperative hospital stay in EOF group was significantly lower than DOF group (5.18 \pm 1.47 days vs $6.18 \pm 2.46$ days, $P=0.016)$. Furthermore, there was a trend for a reduction in the time of first flatus $(10.3 \mathrm{hrs})$ and defecation $(12.7 \mathrm{hrs})$ in EOF group compared to DOF group, but it was not statistically significant. Meanwhile, there were no significant differences in postoperative complications between two groups. One patient in the EOF group developed a fistula in the surgical remnant, which was recorded as other leakages; there was no difference between the two groups $(P=0.582)$.

Conclusion: EOF does not seem to be more harmful than DOF, and might significantly improve the short-term outcomes for patients receiving TLRG.

Keywords: gastric cancer, laparoscopic gastrectomy, early oral feeding, postoperative fistula, anastomotic leakage, enhanced recovery after surgery, ERAS

\section{Introduction}

Gastric cancer is the third most common cause of cancer-related deaths globally, and it is the fifth most common cancer worldwide. ${ }^{1}$ It is well-known that surgery is the only potentially curative treatment for patients with gastric carcinoma. However, a patient with complete resection of primary tumors by a standard D2 lymphadenectomy still faces poor clinical outcomes, especially those with advanced gastric cancer. $^{2}$

With the development of fast track surgery and enhanced recovery after surgery (ERAS) programs, ${ }^{3}$ there has been an increasing trend toward minimally invasive procedures and improved quality of life (QOL) following operation. ${ }^{4}$ Early oral 
feeding (EOF) is generally regarded as one of the fundamental tenets of programs in ERAS, and it has been shown to have beneficial clinical outcomes. ${ }^{5}$

EOF was confirmed as a safe and feasible procedure that increased clinical benefits in the perioperative care of colorectal neoplasm resections. ${ }^{6,7}$ A systematic review and metaanalysis showed that EOF was a beneficial procedure after gastrectomy, as well as operations on hepato-biliary and operations on pancreatic. ${ }^{8}$ Hur et $\mathrm{al}^{9}$ concluded that EOF following a gastrectomy was feasible, and it could result in a faster recovery of bowel function and a shorter hospitalization. The same conclusions were also reached in their subsequent prospective randomized controlled trial (RCT) that assessed the feasibility of EOF in 54 participants undergoing an open gastrectomy. Meanwhile, additional studies ${ }^{10}$ on these topics have also shown that EOF is a safe and feasible program that could shorten the length of hospital stay and improve QOL during the early period following gastrectomy, including faster recovery of bowel function. Furthermore, the meta-analysis that included participants with an upper gastrointestinal surgery showed that postoperative EOF had advantages in the hospital length of stay and when compared to late oral feeding. EOF was beneficial to hospital length of stay, and there were no significant differences in clinically relevant complications. ${ }^{8}$ The guideline for enhanced recovery after gastrectomy ultimately gives weak recommendation for EOF after gastrectomy with a moderate quality of evidence. ${ }^{3}$

Our previous retrospective study ${ }^{11}$ also indicated that EOF could have short-term benefits for patients undergoing laparoscopic distal gastrectomy. However, until now, there has been a lack of RCT on EOF for patients undergoing total laparoscopic radical gastrectomy (TLRG). Thus, a prospective randomized trial is necessary to provide robust evidence on TLRG. This study had been registered online in the Chinese Clinical Trial Registry (ChiCTR-IOR-15007660). The development of this manuscript rigorously followed the standards set by the CONSORT statement. ${ }^{12}$

\section{Materials and methods Study design and participant characteristics}

This study was a prospective, single center, noninferiority, RCT. The number randomization was computer-generated, and the sequence was placed in a sealed envelope by an individual not involved in this trial. If the inclusion criteria were fulfilled, the participants were randomly allocated (1:1) to the EOF group or delayed oral feeding (DOF) group on the morning of postoperative day (POD) 1. The interventions in this study were not blinded for the participants.

There was a total of 100 patients involved from August 27, 2015 to March 31, 2017. They were all diagnosed with gastric cancer and were undergoing a TLRG. Diagnosis and tumor stages were established before surgery through gastroscope and biopsies. We excluded participants with local extension or other distal metastases determined by computed tomography with intravenous contrast of both the abdomen and thorax.

The inclusion criteria were as follows: 1) age range of 18-75 years; 2) diagnosis of gastric cancer and eligible for laparoscopic radical gastrectomy based on the 14th edition of the Japanese Classification of Gastric Carcinoma; 3) no chemotherapy, radiotherapy, or targeted therapy before surgery; 4) an ECOG performance status $<2$; 5) the American Society of Anesthesiologist Physical Status Classification System is 1 or 2 ; 6) the Nutritional Risk Screening $2002(\text { NRS2002) })^{13} \leq 5$; and 7) provide informed consent.

Data were assessed according to the rules of the ethical board at Xijing hospital, and informed consent was acknowledged for each participant and the patient consent was written informed consent. The entire study was approved by the ethical committee at Xijing Hospital of Fourth Military Medical University, which conducted in accordance with the Declaration of Helsinki.

\section{Perioperative treatment}

We rigorously followed the ERAS guidelines. ${ }^{3}$ All participants received preoperative education, pre-emptive and multimodal analgesia, early ambulation, and laparoscopic radical gastrectomy as part of the perioperative treatments.

All procedures of laparoscopic radical gastrectomy were performed by the same experienced surgical teams that had an annual caseload of approximately 100 patients receiving TLRG. In the present study, the procedure of TLRG was defined that a standard D2 lymphadenectomy and the main digestive tract anastomoses (gastrojejunostomy, esophageal-jejunostomy or gastroduodenal anastomosis) were performed completely laparoscopically, instead of an open or laparoscopic-assisted radical gastrectomy (LARG). Moreover, the length of the abdominal incision must be $<10 \mathrm{~cm}$ in this study, which was referenced from the study of CLASS- $0 .{ }^{14}$ An abdominal drain was routinely placed near the anastomosis. The nasogastric 
tube was removed on the morning of POD 1. However, if necessary, a nasojejunal tube was alternatively placed for some patients with a poor nutrition status in both groups to ensure adequate caloric intake after surgery. An investigator was assigned to record the clinical observation data.

\section{Interventions}

All 100 participants were treated similarly in the perioperative period, with the exception of the time of oral feeding after surgery (EOF and DOF group). The criteria for discharge were as follows: tolerance of solid diet, return of bowel habits, and no sign of a postoperative complication that required treatment in the hospital. There was a senior surgeon who was not involved in the study that managed the discharge administration to avoid subjective influences on the trial outcomes.

\section{Early oral feeding (EOF group)}

In the EOF group, participants were encouraged to consume liquid food on the morning of the POD 1, which was based on the registered protocol. Generally, patients were allowed to have pure liquids like glucose in normal saline before regular oral feeding. The volume of EOF ranged from 50 to $500 \mathrm{~mL}$ and was given several times a day. The investigators encouraged EOF patients to take as much as they could tolerate and then recorded it in detail. From POD 2 to 6, liquid foods, such as standard enteral nutrition (EN) liquids or milk, were mainly used for perioperative nutrition management. Additionally, semi-liquids, such as rice soup, eggs, and soft cakes, were also adopted according to the different tolerances of different individuals. Parenteral nutrition (PN), which comprised of fat emulsion, amino acids, and glucose, was used to supply adequate kilocalories (kcal). This was especially true for patients with a poor nutrition status and those that could not consume food orally due to postoperative complications, such as anastomotic leakage. PN was terminated as soon as EN was well tolerated and they could meet the required kilocalories (via oral feeding or nasojejunal feeding tube). We calculated caloric requirements using the Harris-Benedict formula for postoperative energy requirements and measured patients' kilocalorie intake per day. PN could provide a total of 1,000-1,500, 800-1,000, and 500-800 kcal at POD 1, POD 2 and POD 3, respectively.

\section{Delayed oral feeding (DOF group)}

In the DOF group, the patients were allowed to consume liquid food on the morning of POD 4, and they were limited to oral feeding from POD 1 to 3 . Whether nasogastric and nasoenteral feeding tubes were used or not, patients in the DOF group were allowed the same oral intake as the EOF group based on the individual nutritional needs after POD 7.

\section{Study endpoints}

The primary endpoint was the incidence of anastomotic leakage after TLRG. If it was clinically suspected postoperatively, digestive tract radiography per oral contrast was performed to diagnose the leak and its location. While the secondary endpoints were 1) postoperative complications at 30 days, which was defined postoperative morbidity as significant morbidity, grades II, III, IV and $\mathrm{V}$ according to the Clavien Dindo classification, ${ }^{15}$ even if wound infection, classified in grade I, was also considered in postoperative morbidity. We also recorded other data including the need for readmission within 30 days, rate of reoperation; 2) postoperative bowel function recovery which measured as the time to first flatus and the time of first defecation, 3) length of hospital stay and 4) hospitalization costs. For patients with two or more complications only the highest grade was reported, while death (Grade V), reoperation (Grade III) and blood transfusion (Grade II) were reported separately.

\section{Statistical analysis}

The intention-to-treat principle was applied in all analyses of an assumed drop-out rate. Normally distributed continuous variables were described as the mean with corresponding SDs, and categorical variables were described as medians with the corresponding range or percentages and frequencies. Normally or non-normally distributed continuous data was compared by Student's $t$-test or the MannWhitney $U$ test, and the chi-square test and Fisher's exact test were used to compare categorical variables. A twosided $P$-value $<0.05$ was used to represent statistical significance. Data analysis was performed using SPSS $^{\circledR}$ software package version 22.0 (IBM Corporation, Armonk, NY, USA).

\section{Results}

From August 2015 to March 2017, a total of 156 patients were assessed for eligibility, and 56 patients were excluded. Ultimately, 100 enrolled patients were randomly allocated to either the EOF group $(n=51)$ or the DOF group $(n=49)$ (Figure 1). The demographic, clinical, 


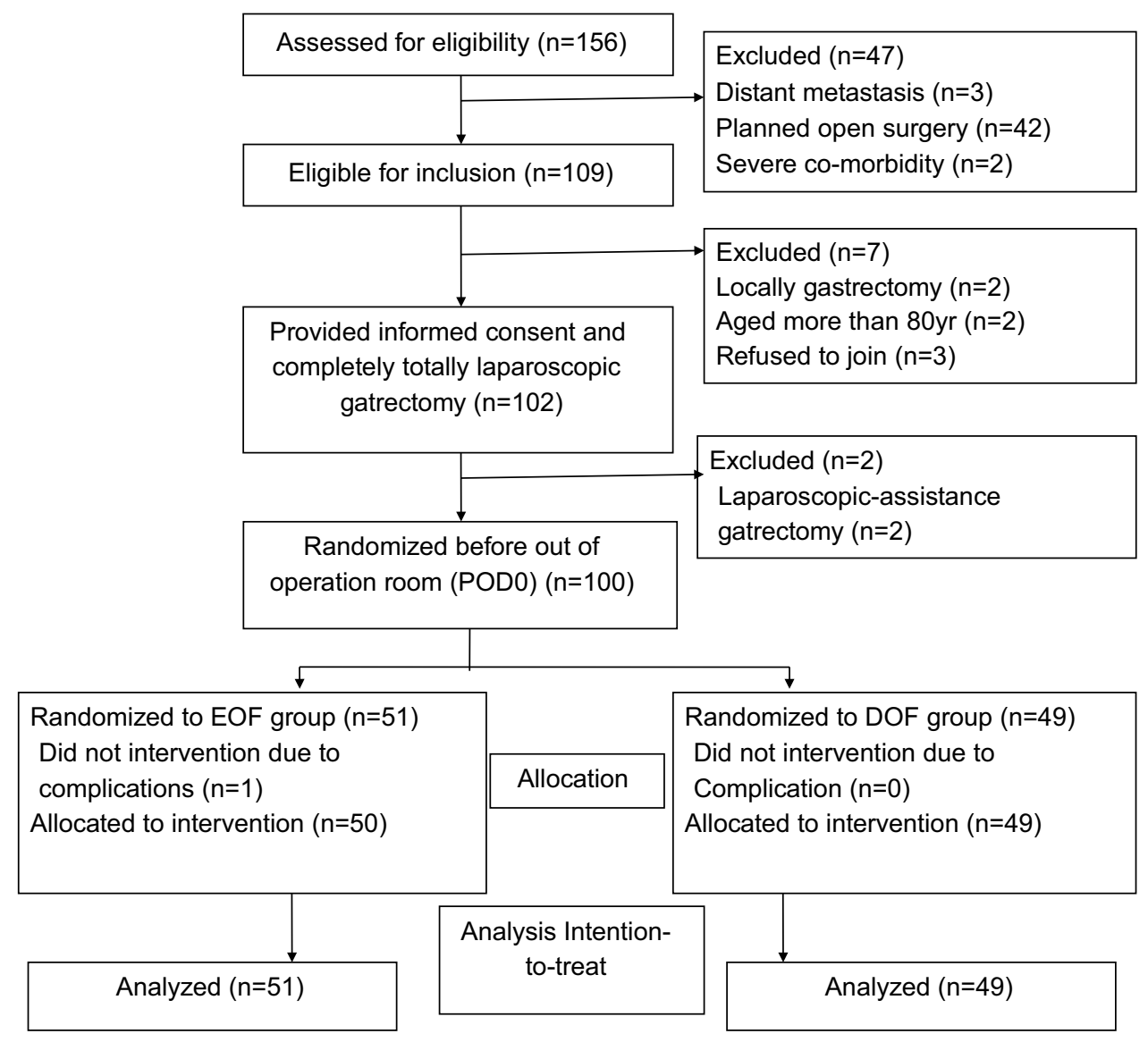

Figure I CONSORT diagram.

Abbreviations: DOF, delayed oral feeding; EOF, early oral feeding; POD, postoperative day.

operative, and pathological characteristics of both groups were all similar at baseline (Table 1).

\section{Study endpoints}

The primary and secondary endpoints were summarized in Tables 2 and 3 respectively. Among 100 patients, there was no anastomotic leakage (zero-event) that occurred in either group within 30 days after TLRG (Table 2). For the outcome of postoperative bowel function recovery, there was a trend of improvement in EOF group, but it was not significant difference ( $P=0.18$ for time of first flatus and 0.12 for time of first defecation) (Table 2), as well as intra-operative data (operative time and blood loss) and hospitalization cost, which did not differ significantly between the two groups. However, EOF group was superior to DOF in the length of postoperative hospital stay $(P=0.016)$.

There was no significant differences in postoperative complications between two groups, such as abdominal bleeding and pulmonary complications. Both groups were no death and no incision infection in the postoperative 30 days, while two patients from EOF group and one from DOF were suffered from the re-operation, and only one patient with anastomotic bleeding and postoperative anemia in EOF group was treated by blood transfusion.

In the EOF group, the data of tolerance volume $(\mathrm{mL})$ at POD 1-3 indicated that the average tolerance volume of oral intake was $206 \mathrm{~mL}$ at POD 1, $490 \mathrm{~mL}$ at POD 2, and $708 \mathrm{~mL}$ at POD 3 (Figure 2).

\section{Discussion}

The current evidence reveals that EOF is a safe and feasible strategy that does not have increased incidences of complications in patients suffering from colonic ${ }^{16}$ and gastric surgery. ${ }^{17}$ Furthermore, animal studies demonstrated that EOF could speed up recovery of peristalsis, strengthen the immune response, ${ }^{18}$ and it could also facilitate anastomotic healing. ${ }^{19,20}$ However, many surgeons were still reluctant to EOF and they preferred to accept traditional restriction after upper gastrointestinal surgery. ${ }^{8}$ 
Table I Clinical data and basic characteristics of patients

\begin{tabular}{|c|c|c|c|}
\hline Characteristic & EOF group $(n=5 I)$ & DOF group $(n=49)$ & $\boldsymbol{P}$ \\
\hline Age (years) & $53.4 I \pm 9.77$ & $55.10 \pm 8.89$ & 0.369 \\
\hline Gendar (M/F) & $37 / 14$ & $34 / 15$ & 0.728 \\
\hline BMI $\left(\mathrm{kg} / \mathrm{m}^{2}\right)$ & $22.58 \pm 3.51$ & $22.96 \pm 3.75$ & 0.580 \\
\hline NRS2002 scores & $2.35 \pm 0.976$ & $2.35 \pm 0.855$ & 0.974 \\
\hline Totally/distal gastrectomy & $4 / 47$ & $5 / 44$ & 0.680 \\
\hline Anastomosis & & & 0.839 \\
\hline Esophagojejunostomy & 4 & 5 & \\
\hline Gastrojejunostomy & 36 & 32 & \\
\hline Gastroduodenostomy & 11 & 12 & \\
\hline Length of abdominal incision $(\mathrm{cm})$ & $6.25 \pm 1.55$ & $6.10 \pm 1.62$ & 0.631 \\
\hline No. of metastatic lymph nodes & $3.22 \pm 5.40$ & $3.57 \pm 4.53$ & 0.721 \\
\hline No. of harvested lymph nodes & $23.14 \pm 5.35$ & $22.82 \pm 5.44$ & 0.767 \\
\hline Differentiation & & & 0.547 \\
\hline Poor & 31 & 31 & \\
\hline Moderate & 11 & 8 & \\
\hline High & 3 & 5 & \\
\hline Other & 6 & 5 & \\
\hline R0 resection & 51 & 44 & I \\
\hline Pathologic stage & & & 0.263 \\
\hline I & 19 & 12 & \\
\hline II & 10 & 15 & \\
\hline III & 22 & 22 & \\
\hline IV & 0 & 0 & \\
\hline
\end{tabular}

Abbreviations: BMI, body mass index; EOF group, early oral feeding group; DOF group, delayed oral feeding group; No., number.

This is because they are mainly concerned with the risk of anastomosis leakage, especially with the absence of sutures to strengthen the anastomotic stoma in laparoscopic surgery. ${ }^{8}$

In the present study, the aim was to illustrate the feasibility of EOF after TLRG regardless of sutures to strengthen the anastomotic stoma. Moreover, it could provide particular data on EOF for patients with TLRG, similar to previously published data on this topic for open or LARG. ${ }^{8}$ Our study demonstrated that, compared to traditional oral intake at POD 4 (DOF group), allowing participants to consume liquid food slowly and cautiously at POD 1 (EOF group) might be safe and feasible, given that the rate of postoperative complications did not differ significantly. There were no anastomosis site-associated adverse events (zero-event) observed in the follow-up period. However, one patient from EOF group, with total laparoscopic radical distal gastrectomy (Billroth II), was clearly diagnosed with a remnant stomach

Table 2 Summary of intraoperative and postoperative data

\begin{tabular}{|l|l|l|l|}
\hline & EOF Group $(\mathrm{n}=5 \mathrm{I})$ & DOF Group $(\mathrm{n}=49)$ & $\boldsymbol{P}$ \\
\hline Intraoperative Data & & & \\
$\quad$ Operative time (min) & $263.53 \pm 78.23$ & $271.73 \pm 83.90$ & 0.614 \\
Blood loss(ml) & $101.33 \pm 69.13$ & $102.04 \pm 54.70$ & 0.955 \\
Postoperative Data & & & \\
Time of first flatus (d) & $2.69 \pm 0.84$ & $3.12 \pm 2.11$ & 0.18 \\
Time of first defecation (d) & $3.71 \pm 1.21$ & $4.24 \pm 2.08$ & 0.12 \\
Drainage tube extraction (d) & $4.04 \pm 1.12$ & $4.22 \pm 1.03$ & 0.39 \\
Postoperative Hospital Stay (d) & $5.18 \pm 1.47$ & $6.18 \pm 2.46$ & 0.016 \\
Costs $\left(10^{4}\right.$ yuan) & $8.24 \pm 1.72$ & $8.44 \pm 1.32$ & 0.492 \\
\hline
\end{tabular}

Abbreviations: EOF group, early oral feeding group; DOF group, delayed oral feeding group. 
Table 3 Summary of postoperative complications

\begin{tabular}{|l|l|l|l|}
\hline & EOF group $(\mathbf{n = 5}$ I) & DOF group (n=49) \\
\hline Complications & & & \\
Anastomotic leakage (n) & 0 & 0 & 1 \\
Others leakages (n) & 2 & 1 & 0.582 \\
Instinal obstruction (n) & 1 & 0 & 0.325 \\
Pulmonary infection (n) & 2 & 0 & $0.16 \mathrm{I}$ \\
Wound infection (n) & 0 & 0 & 1 \\
Abdominal infection (n) & 2 & 1 & 0.582 \\
Gastroparesis (n) & 1 & 2 & 0.534 \\
Anastomotic bleeding (n) & $\mathrm{I}$ & 0 & 0.325 \\
\hline
\end{tabular}

Abbreviations: EOF, early oral feeding; DOF, delayed oral feeding.

fistula at POD 3 through digestive tract radiography per oral contrast, which was proven to locate on the lesser curve near the cardia of the stomach, and excluded gastro-jejunostomysited anastomotic leakage. And it might be regarded as an adverse event of Energy Devices through rechecking the surgical video and the test of gastroscopy. Thus, it was recorded as the number of other leakages from postoperative complications $(n)$. At the meantime, there were no death and incision infection in the following month, and among three patients with re-operations, two patients (one in EOF and 1 in DOF) were diagnosed with postoperative duodenal stump leakage at POD 3 and finally inserted drainage tube to duodenal stump through the operation, while one patient in EOF, diagnosed with severe intestinal obstruction at POD 3, was performed operation again to decompression, and all had recovery in the follow-up. Additionally, only one patient in EOF group was treated by blood transfusion due to anastomotic bleeding and postoperative anemia.

On the other hand, published studies have shown that EOF might improve the recovery of gut function. In the present trial, although there was no significant difference between two groups for time of first flatus $(P=0.18)$ and time of first defecation $(P=0.12)$, a trend in EOF was observed as reduction with an MD (mean difference) of 0.43 day (10.3 hrs) and 0.53 day (12.7 hrs), respectively. And we failed to record these outcomes by usage of hours (hr), which might be considered to be more precise. These findings are similar to our previously retrospective

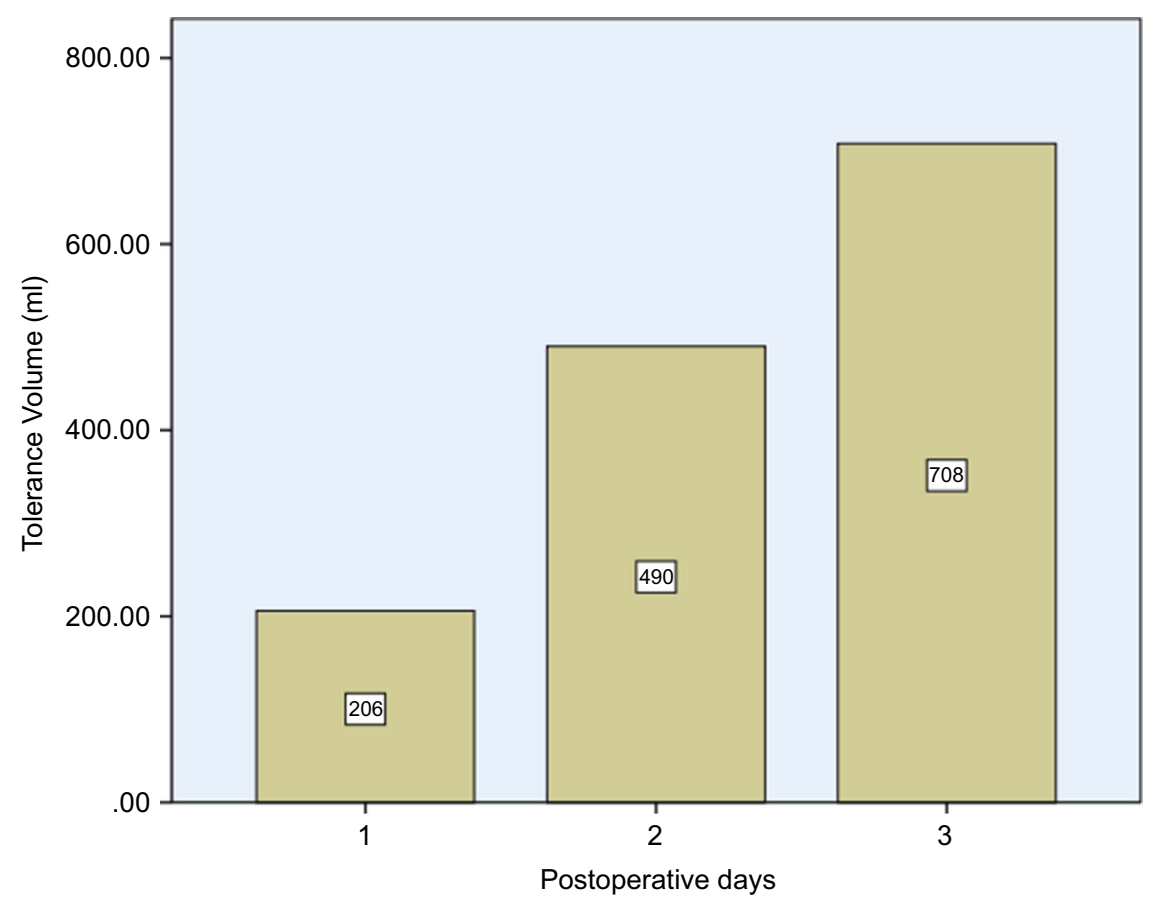

Figure 2 The tolerance volume in EOF group at postoperative days I-3. Abbreviation: EOF, early oral feeding. 
study ${ }^{11}$ and other studies. ${ }^{5,17}$ However, the interpretation of a shorter hospital stay in the EOF group was mainly the well-tolerated liquid or soft food, which might be easier to meet the discharge criteria, such as tolerance of solid diet, return of bowel habits and ability to walk on their own. ${ }^{21}$

The data from the tolerance volume $(\mathrm{mL})$ at POD 1 to 3 of the EOF group of 51 patients indicated that most patients undergoing TLRG could tolerate relatively adequate volumes. This could provide data on the tolerance volume of early oral intake after laparoscopic gastrectomy. Only two participants $(2 / 53)$ did not tolerate any liquids on POD 1, which did not meet the inclusion criteria and they were excluded from EOF groups.

\section{Limitations}

Firstly, we failed to calculate sample size. Our sample size was not large enough to have a robust effect. Moreover, the inadequate number of participants led to a relatively low incidence of morbidity, resulting in zero-event for both groups. Secondly, the age of all included participants in our study was limited to a range of $18-75$ years. This results in a scarcity of evidence for elder patients (>75 years) and patients with a history of neoadjuvant therapy, which had been clearly excluded in study protocol. Thirdly, there were three different anastomoses in total adopted in the trial and it is likely to fail a confirmed conclusion in the pretty small number of cases. Obviously, all limitations above restricted the evidence that supports the popularization of EOF, and further studies are needed to fully assess the feasibility of EOF for patients with worse conditions.

\section{Conclusions}

EOF after total laparoscopic gastrectomy might be a safe and feasible strategy in high volume hospital. However, our findings should be confirmed by further studies to generate prudent and robust evidence. Before it could be routinely advocated in clinical practice, more multi-center, prospective, large sample size RCTs on this topic are urgently required.

\section{Acknowledgments}

This work was supported by Wu Jie-Ping Medical Foundation (320.6750.17508) and Open fund of the Key Laboratory of Evidence Based Medicine and Knowledge Translation of Gansu Province (GSKL-EBM\&KT-
201901). We thank the assistance of a MogoEdit to improve the quality of language in our manuscript.

\section{Author contributions}

QW, ZDY, DZ, LFS and GJ, as the main surgeons, performed the operations. QW, KLY, JY, DZ, and BYG were the main investigators and data recorders. KLY and JY tested the feasibility of the study. QW, KLY and GJ wrote the manuscript. All authors contributed to the research design, drafting and revising the manuscript, gave final approval of the version to be published, and agree to be accountable for all aspects of the work.

\section{Disclosure}

The authors report no conflicts of interest in this work.

\section{References}

1. Torre LA, Bray F, Siegel RL, Ferlay J, Lortet-Tieulent J, Jemal A. Global cancer statistics, 2012. CA Cancer J Clin. 2015 Mar;65 (2):87-108. doi:10.3322/caac.21262

2. Tinoco RC, Tinoco AC, Elkadre LJ, Sueth DM, Conde LM. Laparoscopic gastrectomy for gastric cancer. Dig Surg. 2013;30:132-141. doi:10.1159/000350884

3. Mortensen K, Nilsson M, Slim K, et al. Consensus guidelines for enhanced recovery after gastrectomy. $\mathrm{Br} \quad J \quad$ Surg. 2014;101:1209-1229. doi:10.1002/bjs.9582

4. Kim AR, Cho J, Hsu YJ, et al. Changes of quality of life in gastric cancer patients after curative resection: a longitudinal cohort study in Korea. Ann Surg. 2012;256:1008. doi:10.1097/SLA.0b013e31827661c9

5. Hur H, Kim S, Shim J, et al. Effect of early oral feeding after gastric cancer surgery: a result of randomized clinical trial. Surgery. 2011;149:561-568. doi:10.1016/j.surg.2010.10.003

6. Petrelli NJ, Cheng C, Driscoll D, Rodriguez-Bigas MA. Early postoperative oral feeding after colectomy: an analysis of factors that may predict failure. Ann Surg Oncol. 2001;8:796. doi:10.1007/s10434001-0796-8

7. Wang ZH, Zhong B, Xiang JY, Zhou YB, Wang DS. [Effect of early oral enteral nutrition on clinical outcomes after colorectal cancer surgery]. Zhonghua Wei Chang Wai Ke Za Zhi. 2013;16:735-738.

8. Willcutts KF, Chung MC, Erenberg CL, Finn KL, Schirmer BD, Byham-Gray LD. Early oral feeding as compared with traditional timing of oral feeding after upper gastrointestinal surgery: a systematic review and meta-analysis. Ann Surg. 2016;264:54-63.

9. Hur H, Si Y, Kang WK, Kim W, Jeon HM. Effects of early oral feeding on surgical outcomes and recovery after curative surgery for gastric cancer: pilot study results. World J Surg. 2009;33:1454-1458. doi:10.1007/s00268-009-0009-3

10. Kim J, Kim W, Cheong JH, Hyung WJ, Choi SH, Noh SH. Safety and efficacy of fast-track surgery in laparoscopic distal gastrectomy for gastric cancer: a randomized clinical trial. World J Surg. 2012;36 (12):2879-2887. doi: 10.1007/s00268-012-1741-7

11. Hong L, Han Y, Zhang H, et al. Effect of early oral feeding on short-term outcome of patients receiving laparoscopic distal gastrectomy: a retrospective cohort study. Int J Surg. 2014;12:637-639. doi:10.1016/j.ijsu.2014.05.062

12. Altman DG, Schulz KF, Moher D, et al. The revised CONSORT statement for reporting randomized trials: explanation and elaboration. The CONSORT Group. Chin JEvid-Based Med. 2001;134:663-694. 
13. Gur AS, Atahan K, Aladag I, et al. The efficacy of Nutrition Risk Screening-2002 (NRS-2002) to decide on the nutritional support in general surgery patients. Bratisl Lek Listy. 2009;110:290.

14. Hu Y, Huang C, Sun Y, et al. Morbidity and mortality of laparoscopic versus open $\mathrm{d} 2$ distal gastrectomy for advanced gastric cancer: a randomized controlled trial. J Clin Oncol. 2016;34:1350-1357. doi:10.1200/JCO.2015.63.7215

15. Dindo D. The Clavien-Dindo Classification of Surgical Complications. London: Springer; 2014.

16. Vlug MS, Wind J, Zaag EVD, Ubbink DT, Cense HA, Bemelman WA. Systematic review of laparoscopic vs open colonic surgery within an enhanced recovery programme. Colorectal Dis. 2010;11:335-343. doi:10.1111/j.1463-1318.2008.01679.x

17. Liu X, Wang D, Zheng L, Mou T, Liu H, Li G. Is early oral feeding after gastric cancer surgery feasible? A systematic review and meta-analysis of randomized controlled trials. PLoS One. 2014;9: e112062. doi:10.1371/journal.pone. 0112062
18. Minig L, Biffi R, Zanagnolo V, et al. Early oral versus "traditional" postoperative feeding in gynecologic oncology patients undergoing intestinal resection: a randomized controlled trial. Ann Surg Oncol. 2009;16:1660-1668. doi:10.1245/s10434-009-0444-2

19. Tadano S, Terashima H, Fukuzawa J, et al. Early postoperative oral intake accelerates upper gastrointestinal anastomotic healing in the rat model. J Surg Res. 2007;31:1234.

20. Tadano S, Terashima H, Fukuzawa J, Matsuo R, Ikeda O, Ohkohchi N. Early postoperative oral intake accelerates upper gastrointestinal anastomotic healing in the rat model. J Surg Res. 2011;169:202-208. doi:10.1016/j.jss.2010.01.004

21. Abdikarim I, Cao X, Li S, Zhao Y, Taupyk Y, Wang Q. Enhanced recovery after surgery with laparoscopic radical gastrectomy for stomach carcinomas. World J Gastroenterol. 2015;21:13339-13344. doi:10.3748/wjg.v21.i47.13339

\section{Publish your work in this journal}

Cancer Management and Research is an international, peer-reviewed open access journal focusing on cancer research and the optimal use of preventative and integrated treatment interventions to achieve improved outcomes, enhanced survival and quality of life for the cancer patient.
The manuscript management system is completely online and includes a very quick and fair peer-review system, which is all easy to use. Visit http://www.dovepress.com/testimonials.php to read real quotes from published authors. 